\title{
Editorial
}

\section{End of Lines and Boxes}

\author{
Roger L. Albin, MD* \\ Department of Neurology, University of Michigan, Geriatrics Research, Education, and Clinical Center, Ann Arbor VAMC, \\ Ann Arbor, Michigan
}

In this issue, Joel publishes a thoughtful iteration of the circuit models that have dominated thinking about basal ganglia pathophysiology for almost 15 years. ${ }^{1-4}$ These models have generally been fruitful in the sense that they have led to new experiments, and somewhat surprisingly, provided support and guidance for novel therapeutic approaches. For example, these models all emphasized the importance of the subthalamic nucleus as a key regulator of basal ganglia output, and provided a good deal of impetus for the implementation of pallidotomy and subthalamic deep brain stimulation as treatments for parkinsonism. The relative success of these models is surprising in view of the fact that they are simple anatomic constructs with little effort to incorporate anything beyond the crudest notions of physiology. Many aspects of these models have been challenged appropriately. As Joel makes clear, the anatomy of the basal ganglia is much more complicated than the original versions of these models present. In particular, Joel takes pains to incorporate advances in knowledge of striatal output pathways that add new dimensions of complexity to basal ganglia circuitry. While the original models incorporated the idea of "closed" cortico-basal ganglionicthalamocortical loops and had simple definitions of the so-called direct and indirect pathways, Joel and others emphasize the interconnection between these circuits. ${ }^{5-7}$ This approach has the virtue, explicated rigorously by Joel, of allowing relatively discrete lesions to have consequences that ramify beyond the major function of the circuit primarily affected. In Joel's construct, many phenomena associated with Huntington disease can be ex-

*Correspondence to: Roger L. Albin, M.D., Neuroscience Laboratory Building, 1103 E. Huron, Ann Arbor, MI 48104-1687. E-mail: ralbin@umich.edu

Received 27 November 2000; Accepted 29 November 2000

Published online 9 May 2001 plained by pathology in the associative portions of the striatum. Joel also points out some predictions of the model that can be evaluated experimentally, a necessary feature of any good model.

Joel's model preserves one of the strongest and worst features of the original models. These models are not physiologically based, but are rather elaborate examples of clinico-pathologic correlation. This is a strong method for attempting to understand the basis of clinical phenomena and has real power in assisting the development of new therapies. This method is limited ultimately by how much physiological meaning we can extract from studying clinical phenomena. An instructive comparison is the study of perception. In terms of really understanding brain function, it seems that study of perceptual processes, particularly visual perception, is farther advanced than any other area of neuroscience. Perceptual neuroscientists benefit from substantial advantages. Unlike motor physiologists, sensory physiologists can correlate neural activity with precisely defined inputs. These inputs can be decomposed into well-understood physical properties, and the distinguished tradition of psychophysics provides useful measurement tools. These tools can be applied to human experiments, animal experiments, and more recently, functional imaging experiments. Very little work on basal ganglia function can claim the relative success of sensory physiology in giving mechanistic accounts of sensory, particularly visual, function. Models of basal ganglia pathophysiology are formulated in the absence of good understanding of normal basal ganglia function(s).

Joel's model pushes the clinical correlation method to its limits. She makes a number of associations between specific deficits in Huntington disease subjects and speculated dysfunctions within defined pathways. These associations, however, are based on constructs with relatively shaky foundations. She incorporates well, for ex- 
ample, the popular idea that the basal ganglia are involved in regulating motor programs. But what is the actual evidence for this idea? What type of experiments would one do to falsify both the general idea and Joel's specific version of this concept? Without better understanding of normal basal ganglia function(s), there is a real danger that the articulation of these models could become an end in itself. As long as these models lead to interesting experiments, they are useful. Joel, to her credit, suggests some interesting experiments that derive from this model. Mink attempted to recast these models in more concretely physiological terms and suggested other potentially useful experimental approaches. ${ }^{8} \mathrm{We}$ need, however, better neurophysiology of normal basal ganglia function(s) to understand disease processes. Otherwise, there is danger that we could end up like medieval Ptolemaic astronomers, endlessly adding epicycles to our model in order to "save the phenomena."

\section{REFERENCES}

1. Alexander GE, et al. Parallel organization of functionally segregated pathways linking basal ganglia and cortex. Ann Rev Neurosci 1986; 9:37-381.

2. Albin RL, Young AB, Penney JB. The functional anatomy of the basal ganglia disorders. Trends Neurosci 1989;12:366-375.

3. DeLong MR. Primate model of movement disorders of basal ganglia disorders. Trends Neurosci 1990;13:281-285.

4. Crossman AR. Primate models of dyskinesia: the experimental approach to the study of basal ganglia-related involuntary movement disorders. Neuroscience 1987;21:1-40.

5. Joel D, Weiner I. The connections of the primate subthalamic nucleus: Indirect pathways and the open-interconnected scheme of basal ganglia-thalamocortical circuitry. Brain Res Rev 1997;23: 62-78.

6. Parent A, et al. Organization of the basal ganglia: the importance of axonal collateralization. Trends Neurosci 2000;23:S20-S27.

7. Haber SN, Fudge JL, McFarland NR. Striatonigrostriatal pathways in primates form an ascending spiral from the shell to the dorsolateral striatum. J Neurosci 2000;20:2369-2382.

8. Mink JW. The basal ganglia: focused selection and inhibition of competing motor programs. Prog Neurobiol 1996;50:381-425. 